\title{
APLICAÇÃO DE CORTICÓIDE INTRATIMPÂNICO EM SURDEZ SÚBITA: UM RELATO DE CASO DE SUCESSO TERAPÊUTICO
}

\section{ARTIGO ORIGINAL}

PASSOS, Fabio Manoel dos ${ }^{1}$

PASSOS, Fabio Manoel dos. Aplicação de Corticóide Intratimpânico em surdez súbita: Um relato de caso de sucesso terapêutico. Revista Científica Multidisciplinar Núcleo do Conhecimento. Ano 05, Ed. 12, Vol. 07, pp. 53-60. Dezembro de 2020. ISSN: 2448-0959, Link de acesso: https://www.nucleodoconhecimento.com.br/saude/aplicacao-de-corticoide

\section{RESUMO}

A surdez súbita é caracterizada pela premência otorrinolaringológica, de caráter neurossensorial, sem fator etiológico definido. O presente artigo tem como objetivo discutir o tratamento de uma paciente portadora de tal prognóstico. Será discutido a perda brusca de audição e os benefícios do tratamento precoce para obter um melhor prognóstico. $\mathrm{O}$ uso de corticoides já está bem sedimentado diante das literaturas e suas vias de administração fortemente estruturadas. $\mathrm{O}$ artigo descreve um caso bemsucedido de tratamento utilizando aplicação intratimpânica de corticoide em uma paciente com surdez súbita.

Palavras-chave: Otologia, surdez súbita, corticoide intratimpânico.

\section{INTRODUÇÃO}

A perda abrupta da audição, pode ser acompanhada por comprometimentos psíquicos, visto ser uma experiência terrível e extremamente incômoda para os pacientes. O silêncio repentino em muitos casos pode ser acompanhado de zumbidos

1 Residente em Otorrinolaringologia e Cirurgia Cérvico-Facial; Graduação em Medicina pela Universidade Federal do Paraná - UFPR. 
e vertigens, o que causa no enfermo uma apreensão, visto que é possível que o mesmo perca definitivamente suas vias auditivas. Acoplado a isso, o médico deve identificar e tratar as dores psicológicas e físicas que o paciente pode sofrer devido a sua condição abrupta de perda auditiva.

Por se tratar de uma urgência otológica o médico, otorrinolaringologista ou clínico geral, deverá, prontamente, instituir o diagnóstico e iniciar precocemente o tratamento, para que se modifique o prognóstico da doença.

\section{RELATO DO CASO}

Paciente, E.S.K, sexo feminino, 31 anos de idade, estudante, diabética insulinodependente, procedente da cidade de Cuiabá-MT. A paciente procurou o pronto atendimento especializado em otorrinolaringologia da cidade de Cuiabá em 11 de outubro de 2019 com queixas de diminuição auditiva na orelha esquerda, associado a isso, a paciente ainda relata que estava sentindo tinnitus e tontura há dois dias.

Diante disso, a paciente foi submetida ao exame físico, a mesma se apresentava em bom estado geral, orientada em tempo e espaço, normocorada, hidratada, eupneica e normotensa. Não apresentava alterações à orofaringoscopia, rinoscopia anterior e otoscopia.

Foram solicitados exames audiométricos, o exame de potenciais evocados auditivos de Tronco Encefálico (PEATE) e a ressonância dos ossos temporais. À audiometria evidenciou uma curva audiométrica assimétrica, limiares auditivos normais em orelha direita e do tipo sensorioneural de grau moderadamente severo a severo com configuração ascendente em orelha esquerda (figura 1). O PEATE não mostrou suspeita de disfunção retrococlear na OD, sendo inconclusivo na orelha esquerda devido à ausência de ondas. A Ressonância Nuclear Magnética de ossos temporais não mostrou alterações. 
Figura 1 - Curva audiométrica assimétrica, limiares auditivos normais em orelha direita e do tipo sensorioneural de grau moderadamente severo a severo com configuração ascendente em orelha esquerda.

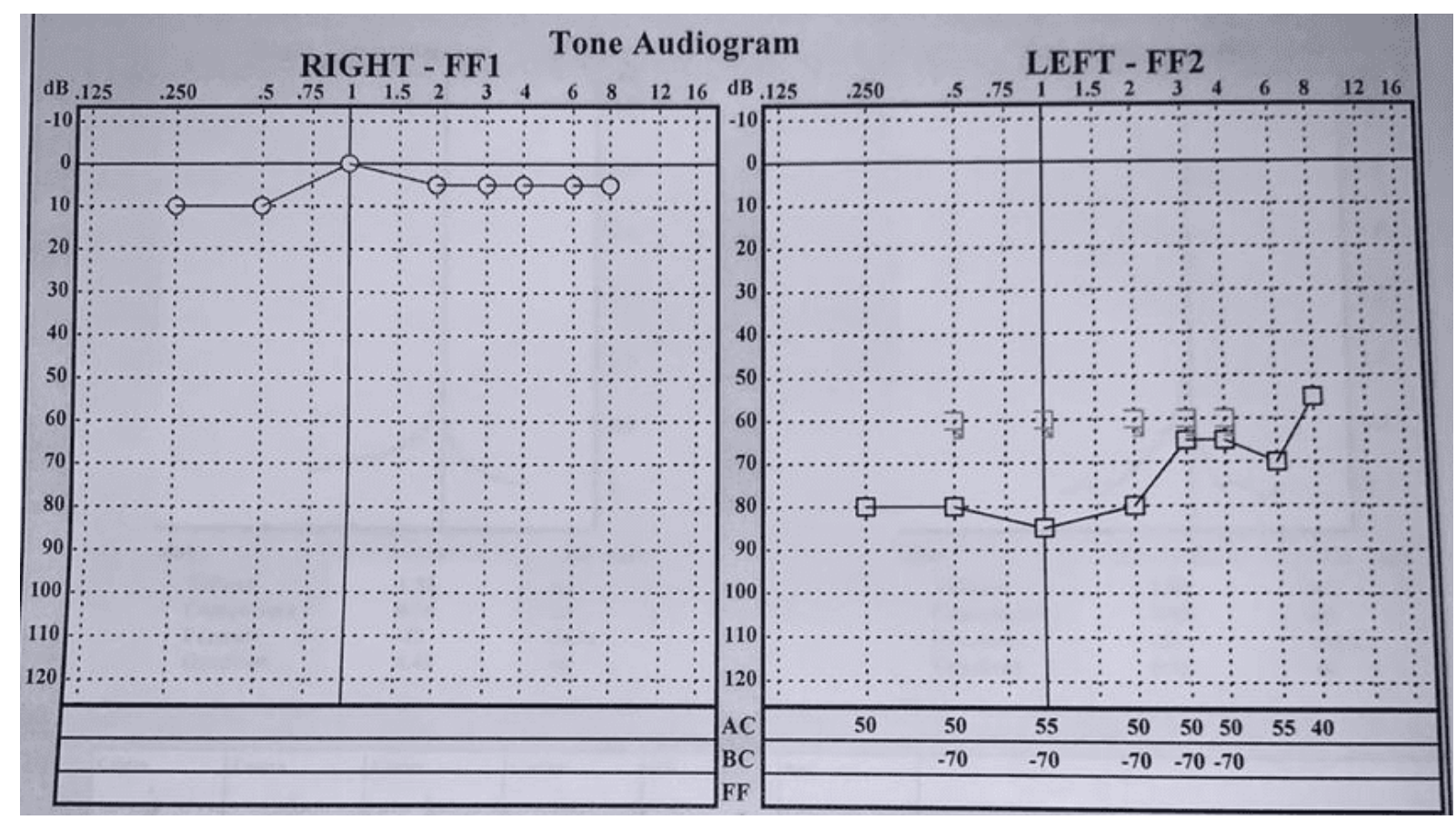

Fonte: compilação do autor.

Discutido com o paciente sobre as opções de tratamento, optamos pela aplicação de corticoide intratimpânico, já que a paciente possuía quadro de diabetes tipo 1 . Realizaram-se duas aplicações de corticoide intratimpânico intercalados por duas semanas e, novamente, foi solicitado que se realizasse a audiometria, novamente, como mostra a figura 2. 
Figura 2 - Curva audiométrica assimétrica, limiares auditivos normais em OD e do tipo sensorioneural, de grau moderado com configuração descendente em rampa na OE.

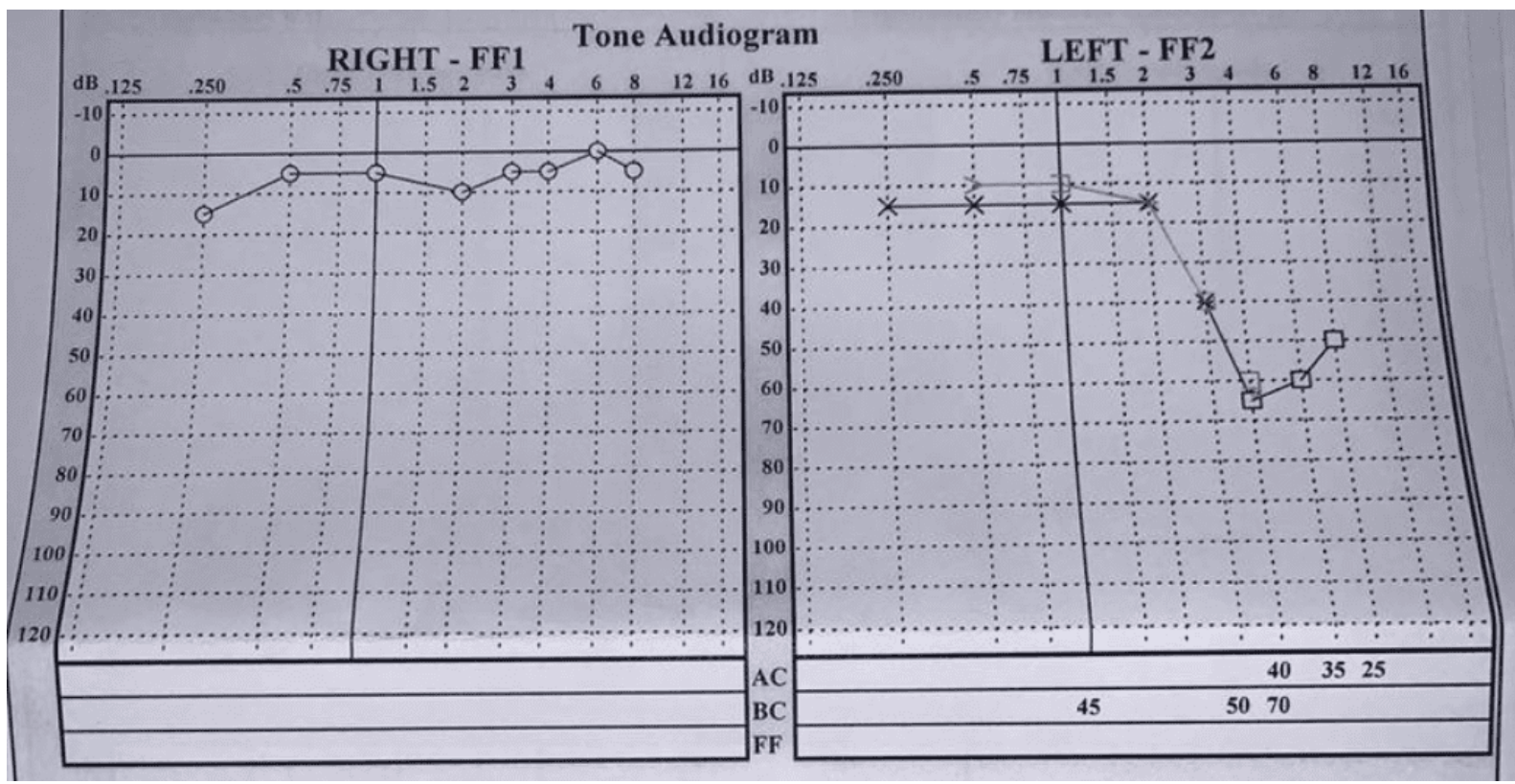

Fonte: compilação do autor.

No retorno, a paciente referia progresso significativo dos sintomas audiométricos. Relatava, também, uma melhora dos sintomas do tinnitus e das vertigens. Devido a isso, foi optado que o seguimento se desse, inicialmente, de forma semestral durante o primeiro ano e continuasse de forma anual, posteriormente.

\section{DISCUSSÃO}

O caso clínico exposto evidenciou uma paciente que de acordo com a história clínica e exames complementares, apresentava diminuição abrupta da audição em orelha direita, com tinnitus associados, leve otalgia com hiperemia leve da membrana timpânica ao exame físico.

De acordo com Penido et al (2014)

Surdez Súbita (SS) é definida como uma perda auditiva maior que $30 \mathrm{~dB}$ em pelo menos três frequências adjacentes de instalação súbita ou em um tempo máximo de até 72 horas, e, na verdade, representa um 
sintoma comum a diversas doenças e não uma entidade nosológica própria.

Se compreende por isso, que a SS, é acometida por muitos, já que a literatura entende que por ano, "a cada 100 mil habitantes, cerca 5 a 20 são acometidos por surdez súbita.", número extremamente significativo. (ALMEIDA; ALMEIDA, 2018)

De acordo com Ben-Hur Santiago Assunção e Carolina Ferreira Marques (2018), "A média de idade do acometimento é de 45,4 anos, sendo mais prevalente na etnia branca. A etiogênese e patogênese são alguns dos aspectos mais estudados, porém continuam sendo alvo de inúmeros questionamentos". A partir desses dados se faz jus considerar o tratamento da surdez súbita, já que essa discussão tem sido uma das questões mais controversas da Otologia.

A literatura compreende por um consenso o uso de corticoides sistêmicos como primeira opção de tratamento, já que essa técnica é tida por estudiosos como sendo o "padrão ouro" de tratamento. Entretanto, o uso de corticoide intratimpânico vem ganhando destaque quando empregado terapeuticamente na segunda linha para tratamento de casos refratários de surdez súbita (RAYMUNDO et al., 2010). Dessa forma, alguns estudos têm mostrado que o uso da terapia como primeira linha é indicado para SS.

A terapia intratimpânica foi relatada inicialmente em 1991, por Itoh, entretanto o tratamento era posto para pacientes portadores da doença de Ménière (ITOH; SAKATA, 1991). O primeiro relato de uso de tal terapia para a SS foi realizada há 24 anos atrás, em 1996, o que a torna recente, por Silverstein, entretanto, seguindo sua perspectiva Slattery, Silverstein, Herr, dentre outros começaram a se utilizar de tal tratamento para a SS (RAYMUNDO et al., 2010). Em 2004, a literatura, passou a discorrer sobre o uso de corticoides intratimpânicos em primeira linha, visando a melhoria da SS, faz jus salientar que o uso de corticoides se faz necessário em casos severos a profundos de perda auditiva (RAYMUNDO et al., 2010).

Raymundo et al. (2010) compreendeu que a janela terapêutica da surdez súbita é curta o que dificultaria a avaliação eficiente dos estudos sobre cada agente, 
isoladamente. Devido a isso, com a ausência de estudos que discorrem sobre a recuperação que se dá devido aos limites do espaço de tempo é difícil determinar a eficácia do tratamento versus a evolução natural da doença, já que não há ainda um paralelo claro traçado dentro dos estudos acerca do tema.

Há alguns artigos que discutem as altas taxas de recuperação espontânea, conquanto, a recuperação auditiva é falha quando não conflui com a corticoterapia sistêmica, sendo este, o motivo pelo qual, no relato de caso se opta pela aplicação do corticoide intratimpânico como primeira opção de tratamento. Ademais, uso de tais corticoides, tem sido indicado, pois evita, em tese, efeitos deletérios secundários do tratamento sistêmico, a exemplo deste: sangramento digestivo; necrose avascular do quadril; aumento da glicose; mudanças de humor; insônia; etc.

Sendo assim, se compreende que o uso da corticoterapia é capaz de atingir em altas concentrações locais, desse modo, aumentaria o efeito nos pacientes afetados. Permeado pelas análises farmacocinéticas, se torna possível a percepção após a aplicação dos corticosteroides no ouvido médio: a concentração na perilinfa segue um modelo de distribuição compartimentada, desta forma, a concentração é perpassada pelo ouvido interno sem difundir para outros compartimentos do corpo, consolidando dessa forma uma ação direta e específica na orelha afetada a ser tratada (GONZÁLEZ; CASTRO, 2007).

Atentando-se ao caso relatado, o uso de corticoides intratimpânicos é considerado a primeira opção de tratamento, devido a administração sistêmica, porque apesar dos riscos, notadamente, a perfuração timpânica persistente, e o uso de intratimpânicos é capaz de reduzir essa incidência. Nessa perspectiva, as perfurações timpânicas que persistem nem sempre estão sendo relatadas dentro da literatura, ainda assim, os artigos que relatam as complicações, perpassam com um percentual de $0 \%$ a $36 \%$. A porcentagem se dá com maior aparência em pacientes com disfunção tubária crônica (GONZÁLEZ; CASTRO, 2007).

Tal tratamento é concluído de vantagens pela literatura: a) o procedimento é bem tolerado e de implementação relativamente fácil, mediante procedimento ambulatorial 
administrado sob anestesia local (tópica); b) pode-se evitar o uso da anestesia geral; c) em sua maioria, os pacientes compreendem o conceito da terapia intratimpânica e aceitam com facilidade o tratamento proposto (HO et al., 2004).

As escolhas dos corticoides se fazem fundamental, a Metilprednisolona foi a medicação selecionada para tratar o caso da paciente que relatamos, o uso se deu devido às propriedades farmacocinéticas e a distribuição no ouvido interno. Ainda assim, trabalhos recentes sugerem, também, o uso da Dexametasona como uma farmacocinética favorável para administração intratimpânica. É sabido que a Dexametasona e a Metilprednisolona possuem potentes efeitos anti-inflamatórios, que, presumivelmente, são relevantes para o tratamento da perda auditiva. Destarte, ambas drogas são concebíveis para prognóstico de eficácia equivalente em dosagem (RAUCH et al., 2011). Sem embargo, é relatado a diferenciação em contraposição à concentração da solução (Dexametasona: 2- $4 \mathrm{mg} / \mathrm{mL}$ a $25 \mathrm{mg} / \mathrm{mL}$; Metilprednisolona $32 \mathrm{mg} / \mathrm{mL}$ a $62.5 \mathrm{mg} / \mathrm{mL}$ ). (RAYMUNDO, 2010)

Um compilado realizado por Igor Teixeira Raymundo et al. (2010) foi capaz de desenvolver e perceber as quantidades $\mathrm{e}$ as formas sob as quais seriam administradas o corticoide. Os autores compreenderam que a quantidade injetada na orelha média varia entre 0.3 e $0.5 \mathrm{~mL}$, sendo, aproximadamente, o volume da orelha média. Os estudiosos entenderam que seria possível aplicar a injeção por intermédio de agulhas: transtimpânica, miringotomia, miringotomia com tubo de ventilação, miringotomia com capilar especial para perfusão (Micromedics, Eagan, MN), e bomba de infusão implantável em orelha média (Round Window m-Cath; Durect Corp., Cupertino, CA) para liberação do esteróide em infusão contínua. A duração do tratamento intervalado entre as injeções e número de injeções, também, varia, pois é vinculado a interdependência da quantidade do corticoide e da via de administração.

No caso apresentado, realizou-se duas aplicações de Metilprednisolona, de $01 \mathrm{~mL}$, por intermédio de injeção intratimpânica, houve intervalo de duas semanas entre elas. Deliberou-se, dessa forma, aplicação intratimpânica do corticoide, como primeira opção de tratamento. Tal decisão foi concebida, visto que a paciente é diabética, desta 
forma a mesma não toleraria os efeitos deletérios da prescrição de altas doses de corticóides sistêmicos.

À vista disso, não se define um protocolo universal aceito para a aplicação de corticosteróides intratimpânicos. Ao perpassar os mais variados estudos, foi possível constatar uma variação no tipo de corticosteróide usado, a concentração de soluções, da quantidade, via de administração, o número de sessões e intervalo entre elas, o que certamente interfere na obtenção de resultados homogêneos.

Contudo, se depreende que a desvantagem da corticoterapia intratimpânica relatada pela literatura médica é, ainda, a falta de comprovação de sua eficácia superior ao ser comparada com a terapia sistêmica. Nada obstante, salta aos olhos em casos específicos, como o relatado anteriormente, a incidência de baixos efeitos colaterais secundários sobretudo, para aqueles grupos de pacientes que não suportariam a administração de altas doses de corticóides via sistêmica, a exemplo dos diabéticos e com hipertensão arterial, o que têm feito alguns profissionais promoverem o seu uso como terapia de primeira linha, indicando-a para todos os casos de Surdez Súbita.

\section{CONCLUSÃO}

A Surdez Súbita é uma condição médica capaz de provocar transtornos e aflições nos pacientes que são acometidos. A perda ou diminuição repentina de um dos sentidos do corpo humano é capaz de produzir danos orgânicos e, também, psicológicos a pessoa afetada, seja no seu ambiente de trabalho ou nas atividades diárias.

A possibilidade de haver um prognóstico desfavorável traz uma sensação de angústia e sofrimento para o paciente. Dessa forma a relação médico paciente deve se pautar na orientação cautelosa, e acompanhamento regular desses casos.

O diagnóstico preciso e precoce diminui o sofrimento do paciente e proporciona uma maior confiança deste no médico e nas opções que o mesmo lhe oferecerá para tratamento, ocorrendo melhor adesão. O tratamento precoce, instituído logo no início dos sintomas, se mostra cada vez mais eficaz e a aplicação intratimpânica de 
corticoide é cada vez mais eficaz, principalmente, na contraindicação de uso e corticóides via oral, sendo uma excelente ferramenta de tratamento adquirindo ótimos resultados.

\section{REFERÊNCIAS}

ALMEIDA, C. I. R.; ALMEIDA, R. R. A. Surdez Súbita. In: Pignatari SSN \& Lima WTA (Org.). Tratado de Otorrinolaringologia. 3. ed. Rio de Janeiro: Elsevier, 2018.

ASSUNÇÃO, B.-H. S.; MARQUES, C. F. Surdez súbita em paciente em uso de tadalafil: um relato de caso clínico. Rev. Ciência Médica e Biológicas, 2018. Disponível em: < https://portalseer.ufba.br/index.php/cmbio/article/view/25152>

BOGAZ, E. A.; et. al. Influência dos corticosteroides no prognóstico auditivo da perda auditiva neurosensorial súbita idiopática. Brasileira j. otorhinolaryngol, 2014. Disponível em: https://www.scielo.br/scielo.php?pid=S180886942014000300213\&script=sci_arttext\&tlng=pt

FARIA, M. B.; et. al. Surdez Súbita - Tratamento com corticoide intratimpânico e oxigenação hiperbárica. Revista Científica Multidisciplinar Núcleo do Conhecimento, 2017. Disponível em: <https://www.nucleodoconhecimento.com.br/saude/surdezsubita?pdf=7305>

GONZÁLEZ, R.; CARO, J. Corticoides intratimpánicos: una revisión sistemática. Rev. Otorrinolaringol. Cir. Cabeza Cuello 2007. Disponível em: < https://pesquisa.bvsalud.org/portal/resource/pt/lil-474883>

GUAN-MIN, H.; et. al. Effectiveness of intratympanic dexamethasone injection in sudden-deafness patients as salvage treatment. 2014. Disponível em: < https://pubmed.ncbi.nlm.nih.gov/15235345/>

ITOH, A.; SAKATA, E. E. Treatment of vestibular disorders. Acta Otolaryngol Suppl. $2009 . \quad$ Disponível em: https://www.tandfonline.com/doi/abs/10.3109/00016489109131486 
LAZARINI, P. R.; CAMARGO, A. C. K. Surdez súbita idiopática: aspectos etiológicos e fisiopatogênicos. Rev. Brasileira Otorrinolaringol, 2006. Disponível em: https://www.scielo.br/scielo.php?pid=S003472992006000400019\&script=sci_abstract\&tlng=pt

PENIDO, N. de O.; et. al. Fatores clínicos, etiológicos e evolutivos da audição na surdez súbita. Rev. Brasileira Otorrinolaringol, 2005. Disponível em: < https://www.scielo.br/scielo.php?pid=S0034-

72992005000500014\&script=sci_abstract\&tlng=pt>

RAYMUNDO, I. T. R.; et. al. Intratympanic Methylprednisolone as Rescue Therapy in Sudden Sensorineural Hearing Loss. Braz J Otorhinolaryngol. 2010. Disponível em: < https://www.scielo.br/pdf/bjorl/v76n4/en_v76n4a15.pdf>

RAUCH, S. D.; et. al. Oral vs intratympanic corticosteroid therapy for idiopathic sudden sensorineural hearing loss. JAMA. 2011. Disponível em: < https://pubmed.ncbi.nlm.nih.gov/21610239/>

Enviado: Novembro, 2020.

Aprovado: Dezembro, 2020. 